Table 2 Number of pancreatin capsules* taken and fat malabsorption (\%) while taking usual and modified pancreatin doses

\begin{tabular}{|c|c|c|c|c|}
\hline \multirow[t]{2}{*}{ Case No } & \multicolumn{2}{|c|}{ Usual pancreatin dose } & \multicolumn{2}{|c|}{ Modified pancreatin dose } \\
\hline & No of capsules & $\%$ Malabsorptiont & No of capsules & $\%$ Malabsorptiont \\
\hline $\begin{array}{l}1 \\
2 \\
3 \\
4 \\
5 \\
6 \\
7\end{array}$ & $\begin{array}{l}39 \\
37 \\
51 \\
45 \\
50 \\
44 \\
46\end{array}$ & $\begin{array}{r}5 \\
16 \\
8 \\
22 \\
7 \\
7 \\
5\end{array}$ & $\begin{array}{l}18 \\
18 \\
20 \\
27 \\
28 \\
19 \\
19\end{array}$ & $\begin{array}{r}6 \\
18 \\
39 \\
43 \\
5 \\
10 \\
6\end{array}$ \\
\hline
\end{tabular}

${ }^{*}$ Capsule numbers refer to an average daily intake.

$\dagger \%$ Malabsorption refers to the percentage of orally ingested fat malabsorbed, as determined by a three day fat balance study.

reductions in their enzyme intake without appreciable alteration in their absorption. Both these patients achieved normal fat absorption with the reduced enzyme dose.

\section{Discussion}

This study has shown that some patients with cystic fibrosis take inappropriately high doses of pancreatic enzymes and that reduction of this high input may be achieved without deterioration in fat absorption. However, two of the patients did have an increase in fat malabsorption when their enzyme dose was decreased. This argues against a policy of setting an arbitrary limit to the enzyme dose, as is frequently suggested at cystic fibrosis clinical meetings. All patients in this study had reached their current dose of enzymes in response to symptoms perceived as due to residual malabsorption This study highlights the risks of assuming that abdominal symptoms in patients with cystic fibrosis are invariably due to pancreatic enzyme insufficiently and therefore will respond to an increase in enzyme treatment; five of the seven subjects had normal absorption despite persisting symptoms.

The major implication of the results of the present study is that objective assessment of nutrient absorption should be routine in patients with cystic fibrosis, particularly those with abdominal symptoms. The best of the currently available tests is the three day faecal fat balance. This procedure is time consuming, prone to large errors if not performed correctly, and rarely welcomed by patients. The results of the present study, however, illustrate the importance of assessing changes in enzyme treatment by direct measurements of nutrient absorption.

In conclusion the present study has shown that some children with cystic fibrosis may be taking inappropriately high doses of pancreatic enzymes because of abdominal symptoms not directly related to exocrine pancreatic insufficiency. It is important to determine whether symptoms are due to fat malabsorption and that increases in enzyme replacement result in improved absorption. Recognition of other potential causes of abdominal symptoms in cystic fibrosis should lead to a more logical approach to the patient with residual symptoms on standard enzyme treatment.

The authors wish to thank Mrs B Crawford who performed the faecal fat assays. This work was supported by a grant from the Royal Children's Hospital Research Foundation. The financial assistance of Organon is gratefully acknowledged. 1 Baxter PS, Dickson JAS, Variend S, Taylor CJ. Intestinal disease in cystic fibrosis. Arch Dis Child 1988;63:1496-7.
Gaskin KJ, Waters DLM, Howman-Giles R, et al. Liver disease and common bile duct stenosis in cystic fibrosis. $N$ Engl $f$ Med 1988;318:340-6.

3 van de Kramer JH, ten Bokkel Huinink H, Weyers HA. Rapid method for the determination of fat in faeces. $\mathcal{f}$ Biol Chem 1949;177:347-55.

\title{
Dexamethasone treatment for congenital adrenal hyperplasia
}

M C Young, I A Hughes

\section{Department of Child Health University of Wales College of Medicine, Cardiff \\ M C Young \\ I A Hughes \\ Correspondence to: Professor IA Hughes,} University Department of Paediatrics, Addenbrooke's Hospital Level E8, Hills Road, Cambridge CB2 200.

Accepted 8 November 1989

\begin{abstract}
Ten patients with congenital adrenal hyperplasia (three males, seven females; aged 12-29 years) had their usual glucocorticoid treatment changed to dexamethasone in three crossover dosage regimens. A starting dose of $5 \mu \mathrm{g} / \mathrm{kg} / \mathrm{day}$ is suggested but as no one dose regimen resulted in adequate control the timing of the dose must be decided for each patient.
\end{abstract}

The optimum glucocorticoid preparation, total daily dose, and dose schedule for the treatment of congenital adrenal hyperplasia remain controversial. ${ }^{1}$ Hydrocortisone is probably the preparation of choice in infancy and childhood, but in adolescents near completion of growth and adults dexamethasone may be a convenient alternative. ${ }^{1-3}$ The potency of dexamethasone in relation to both pituitary-adrenal suppression and side effects is much greater.

\section{Patients}

Ten patients (three males and seven females) aged 12-29 years with 21-hydroxylase deficiency (classic salt losing type) congenital adrenal hyperplasia took part in the study. 
Patients had been previously treated with multiple daily doses of hydrocortisone and were changed to dexamethasone when growth was complete or because there was difficulty in achieving adequate control (this was found mainly in postmenarchal girls in whom menses were irregular). Each patient had an adult bone age.

Profiles of $170 \mathrm{H}$-progesterone concentrations in blood spot and saliva samples collected at $0800,1200,1800,2200$ hours on two consecutive days, and a single measurement of plasma testosterone concentration at 0900 hours (females only), were used to assess the degree of therapeutic control immediately before starting dexamethasone, $10 \mu \mathrm{g} / \mathrm{kg} /$ day. The total amount was given orally at 0900 for one month, at 2100 hours for one month, and in equally divided doses at 0900 and 2100 hours during the third month of the study. The steroid profiles were repeated at the end of each one month period and the results compared with previously published reference values for adequacy of control. ${ }^{4}$

Capsules containing $100 \mu \mathrm{g}$ of dexamethasone were used to achieve accurate dosage. Patients thereafter reverted to a single morning dose of dexamethasone with the total daily dose adjusted according to the results of regular steroid profiles determined during the ensuing year.

\section{Results}

The degree of therapeutic control achieved during each treatment regimen is shown in the

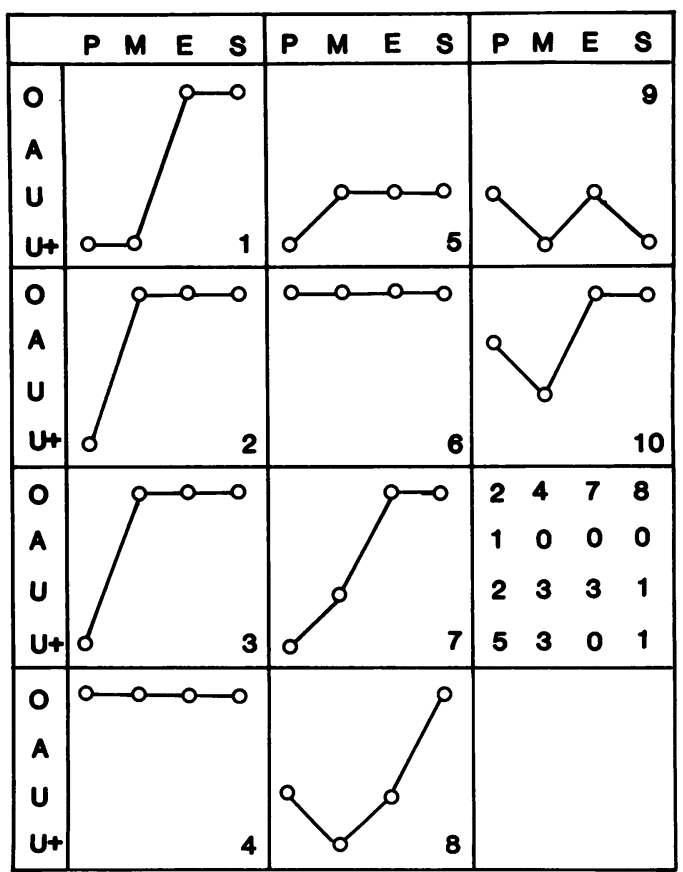

The degree of therapeutic control in 10 patients with congenital adrenal hyperplasia. Control was scored as: $O=$ overtreated; $A=$ adequately treated; $U=$ undertreated; and $U+=$ considerably undertreated. The treatment regimens were: $P=$ previous hydrocortisone treatment; $M=$ morning dose of dexamethasone alone; $E=$ evening dose alone; and $S=$ split dose of dexamethasone (morning and evening). The results in 10 patients are depicted in boxes 1-10; a summary of the entire group is shown in the final box. figure. Six patients were undertreated while on hydrocortisone. The individual patient scores suggested an increased tendency to overtreatment with dexamethasone, particularly when the dexamethasone dose was given in the evening and as a split daily dose. No one dose regimen appeared to result in adequate treatment.

The long term use of the single morning dexamethasone dose required a reduction in the total daily dose (to $<10 \mu \mathrm{g} / \mathrm{kg} /$ day) in five patients and an increase in the dose in one patient before adequate therapeutic control was achieved. All patients reported an increased appetite during this period of stabilisation, and four gained an abnormal amount of weight. An additional patient developed skin striae and mild hirsutism. The final dose requirement of dexamethasone ranged from $0.2-0.75 \mathrm{mg} / \mathrm{day}$; nine patients needed $<0.5 \mathrm{mg} /$ day to ensure adequate control.

\section{Discussion}

Dexamethasone is a synthetic glucocorticoid whose increased potency and longer half life offer the advantages of superior compliance and regulation of menses in older adolescent and adult women with congenital adrenal hyperplasia. Possible growth inhibiting effects are not relevant at this age. Doses which range between $0.25-0.75 \mathrm{mg} /$ day are usually adequate. ${ }^{1-3}$ In this study, $10 \mu \mathrm{g} / \mathrm{kg} /$ day of dexamethasone produced a dose range of $0.35-0.8 \mathrm{mg} /$ day. When dexamethasone was started at this dose, given initially in the morning, there was no significant improvement in therapeutic control. There was also a considerable patient idiosyncracy. More patients appeared to be overtreated, particularly when the dexamethasone was given in the evening (either as a single or split dose). A cumulative effect of the steroid may have been achieved by this stage of the study. The evening administration of glucocorticoid is considered to be more potent because of greater suppression of early morning adrenal steroid concentrations, although dose division and time appear to be unimportant factors when hydrocortisone is used. ${ }^{5}$ Dexamethasone has been successful in achieving adequate control when used either once or twice daily. ${ }^{2} 3$ The need to reduce total daily dose due to cumulative effect, and the minor side effects of dexamethasone have been noted previously. ${ }^{2}$

A change to dexamethasone treatment when growth is complete often leads to improved ovarian function in women and offers an easier option for compliance in all patients with congenital adrenal hyperplasia. We suggest a starting dose of $5 \mu \mathrm{g} / \mathrm{kg} /$ day and it may be necessary to use especially prepared $100 \mu \mathrm{g}$ capsules. The dose should be adjusted subsequently in increments not greater than $100 \mu \mathrm{g}$ according to serial biochiemical parameters of therapeutic control. ${ }^{4}$ The choice of dose division and timing remains a matter for each individual patient, but the possible effects of changes in dose timing on therapeutic control must always be considered. 
1 Hughes IA. Management of congenital adrenal hyperplasia. Arch Dis Child 1988;63:1399-404.

2 Horrocks PM, London DR. Effects of long term dexamethasone treatment in adult patients with congenital adrenal hyperplasia. Clin Endocrinol 1987;27:635-42.

3 Hayek A, Crawford JD, Bode HH. Single dose dexamethasone in treatment of congenital adrenocortical hyperplasia. Metabolism 1971;20:897-901.
4 Young MC, Robinson JA, Read GF, Riad-Fahmy D, Hughes IA. $170 \mathrm{H}$-progesterone rhythms in congenital adrena hyperplasia. Arch Dis Child 1988;63:617-23.

5 Winterer J, Chrousos GP, Loriaux DL, Cutler GB. Effect of hydrocortisone dose schedule on adrenal steroid secretion in congenital adrenal hyperplasia. Ann NY Acad Sci 1985; 458:182-92.

\title{
Sialic acid storage disease
}

\author{
P D Cameron, V Dubowitz, G T N Besley, A H Fensom
}

\begin{abstract}
A baby girl with coarse facial features, hepatosplenomegaly, and developmental delay had raised free sialic acid concentrations in her urine and cultured fibroblasts. She died aged 13 months. Sialic acid is an important constituent of many glycoproteins and glycolipids; impaired release from the lysosome may be the underlying biochemical defect.
\end{abstract}

Disorders of sialic acid metabolism fall into four categories. Raised concentrations of bound sialic acid are found in conditions associated with neuraminidase deficiency. ${ }^{1}$ No enzyme deficiency has been shown in three other categories where raised concentrations of free sialic acid are found: Salla disease, sialuria, and severe infantile sialic acid storage disease. Salla disease, named after an area in northern Finland, comprises progessive psychomotor retardation with lysosomal storage in patients reaching adult life. ${ }^{2}$ Two cases of sialuria have been described where only sialic acid in the urine was raised. These patients had coarse facies, hepatosplenomegaly, a mild clinical course, and no lysosomal storage. ${ }^{3}$ This case report concerns the fourth category: severe infantile sialic acid storage disease.

\section{Case report}

The baby girl was the second child of healthy unrelated white parents; her brother, aged 2 years, was normal. Pregnancy was complicated by premature labour at 32 weeks' gestation; breech presentation necessitated a caesarean section. Birth weight and head circumference were both below the third centile. Resuscitation with intubation was required for five minutes. Initial problems included transient tachypnoea and episodes of cardiac failure with dusky spells and hepatomegaly. Chest radiographs showed cardiomegaly and pulmonary plethora. Transfusion was required for anaemia.

Dysmorphic features comprised hypertelorism, prominent epicanthic folds, strikingly fluffy eyebrows, a long philtrum, and a high arched palate (figure). Facial features were generally coarse. She had short stubby fingers and a square set thumb. Her skin was pale with wispy orange hair.

Diuretics and fluid restriction controlled her

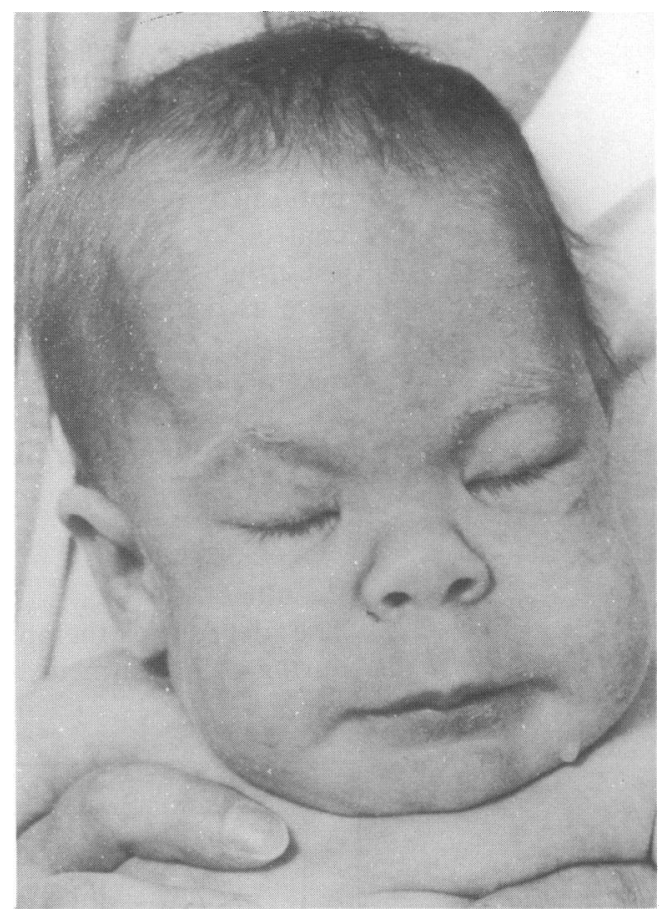

Dysmorphic features showing coarse facies, wispy hair and eyebrows, and telangiectasias.

cardiac failure and she was discharged home at 8 weeks. She was readmitted at 11,13 , and 16 weeks with chest infections and cardiac failure. Hepatosplenomegaly and telangiectasias had become prominent. She developed a need for supplemental oxygen, and weight gain remained poor. By 5 months she had rarely smiled, she remained hypotonic, visual following was poor, and auditory response was absent.

Laboratory investigations at 5 months included measurement of blood concentrations of urea, electrolytes, and haemoglobin, liver function, blood and urine amino acids, urine mucopolysaccharides, and lactate. Chromosome analysis, iron studies, thyroid function tests, and lysosomal enzyme activities were also performed. All studies gave normal results. Skeletal survey, cranial ultrasound, and nitrogen washout tests were also normal. Chest radiography showed fine hazy shadowing in the lung fields and cardiomegaly. Ophthalmic examination showed an intermittent divergent strabis- 